\title{
Effects of Hydroperoxide in Lipid Peroxidation on Dough Fermentation
}

\author{
Toshiyuki Toyosaki
}

Additional information is available at the end of the chapter

http://dx.doi.org/10.5772/39187

\section{Introduction}

The oxidation of lipids in foods is responsible for the formation of off-flavors and chemical compounds that may be detrimental to health; it is a well known problem in the food chemistry and biochemistry fields (Ames et al., 1994; Gardnaer, 1996; Grosch, 1987; Pokorny, 1999; Shewfelt \& Del Rosario, 2000; Mercier \& Gelinas, 2001; Toyosaki \& Sakane, 2002; Toyosaki \& Koketsu, 2004). Currently, the various lipid peroxides produced by such lipid peroxidation are treated only as a nuisance. However, among longstanding traditional foods there are foods with fine flavors that are brought out by inducing lipid peroxidation; such foods include fine, thin noodles and certain dried foods. Thus, lipid peroxides produced by lipid peroxidation can also be advantageous. The properties of foods can be improved by better use of the properties of lipid peroxides. The current work provided an interesting finding: when lipoxygenase was added during the fermentation of bread dough, the fermentation of dough was promoted. During this event, hydroperoxides produced by lipid peroxidation triggered the promotion of fermentation and promoted the fermentation of dough. The phenomenon by which hydroperoxides are produced by lipid peroxidation and promote the fermentation of bread dough is decidedly not beneficial when assessed from a nutritional standpoint, but this phenomenon is extremely desirable when assessed from a food science standpoint. The objective of the current study was to investigate the bread dough fermentation-promoting action of hydroperoxides produced by lipid peroxidation in bread dough and the mechanism that is involved.

\section{Methods}

\section{Preparation of dough}

The wheat flour (strong flour) that was used to make adjustments in the bread dough was the type that is readily commercially available. The lipid added was linoleic acid (more than 
95\% pure), which was added at a 3\% level. Other ingredients used to make the bread were all commercially available. Lipoxygenase was added at the end of bread dough adjustment and underwent primary fermentation in an incubator at $37^{\circ} \mathrm{C}$ with $75-80 \%$ humidity. After fermentation, gas was released; after a bench time of $10 \mathrm{~min}$, the dough underwent final fermentation for $90 \mathrm{~min}$ and was then baked for $12 \mathrm{~min}$ at $200^{\circ} \mathrm{C}$.

\section{Preparation of the model system of gluten and linoleic acid}

By mixing a fixed amount of commercially available gluten and linoleic acid (more than 95\% pure) of $3 \%$ level, which served as the test sample, a model system was created. This sample underwent fermentation by lipoxygenase induction.

\section{Measurement of the rate of dough expansion}

To determine the rate of dough expansion with fermentation, a fixed amount of dough was placed in a graduated cylinder and fermented in an incubator (temperature $30^{\circ} \mathrm{C}$, humidity $75 \%$ ). The rate of expansion over a fixed period of time was then measured.

\section{Measurement of hydroperoxide}

Hydroperoxide concentration was calculated in terms of $2^{\prime}, 7^{\prime}$-dichlorofluorescein (DCF). To de-emulsify, the $5.0 \mathrm{ml}$ samples were centrifuged $(10,000 \times \mathrm{g}$, $30 \mathrm{~min})$. The linoleic acid of the supernatant was then measured to determine the hydroperoxide level using the method of Cathcart et al. (1984). First, $1.0 \mathrm{ml}$ of a $1.0 \mathrm{mM}$ solution of DCF in ethanol and $2.0 \mathrm{ml}$ of $0.01 \mathrm{~N} \mathrm{NaOH}$ were mixed and stirred for $30 \mathrm{~min}$ before being neutralized with $10 \mathrm{ml}$ of 25 $\mathrm{mM}$ phosphate buffer ( $\mathrm{pH} 7.2$ ). Then $2.0 \mathrm{ml}$ of the neutralized DCF solution were added to a solution of hematin $(10 \mathrm{mM})$ in $25 \mathrm{mM}$ phosphate buffer $(\mathrm{pH} 7.2 ; 0.01 \mathrm{mg} \mathrm{DCF} / \mathrm{ml})$; subsequently, $2.0 \mathrm{ml}$ of this hematin-DCF solution and $10 \mathrm{ml}$ of the linoleic acid sample were mixed and left at $50^{\circ} \mathrm{C}$ for $50 \mathrm{~min}$, before fluorometry treatment (excitation. $400 \mathrm{~nm}$; emission. $470 \mathrm{~nm}$ ) to measure DCF. This method measures hydroperoxide with more sensitivity than the iron rhodanide method that is usually used.

\section{Diethylaminoethyl (DEAE) column chromatography}

The extracted dough was separated by Tris- $\mathrm{HCl}$ buffer $(\mathrm{pH} \mathrm{8.0)}$. The extracted sample fractions were separated by DEAE-cellulose (DE52, Whatman, Ltd., Tokyo, Japan) column chromatography as follows. A DEAE-cellulose column $(3.8 \times 54 \mathrm{~cm})$ was equilibrated with $50 \mathrm{mM}$ Tris- $\mathrm{HCl}$ buffer ( $\mathrm{pH} 8.0$ ), washed with the same buffer, and developed in a linear gradient made with $300 \mathrm{ml}$ of this buffer and $300 \mathrm{ml}$ of the same buffer containing $0.6 \mathrm{M}$ $\mathrm{NaCl}$. The flow rate was $30 \mathrm{ml} / \mathrm{hr}$, and $3.0 \mathrm{ml}$ fractions were collected.

\section{Measurement of the amount of protein}

The amount of protein was measured using the Lowry method (1951).

\section{SDS-polyacrylamide gel electrophoresis (PAGE)}

The measurement was done according to the method of Laemmli (1979). Electrophoresis was performed using the Mini-Protean II Electrophoresis Cell (Bio-Rad Laboratories, Inc., 
Tokyo, Japan) at $18 \mathrm{~mA} /$ gel with Ready Gel J of differing gel concentrations. After electrophoresis, the gels were stained using Coomassie brilliant blue-R250. In addition, automated electrophoresis (Phast System; Pharmacia LKB, Biotechnology AB, Uppsala, Sweden) equipment was used.

\section{Statistical analysis}

Analysis of variance (ANOVA) was performed and mean comparisons were obtained by Duncan's multiple range test (Steel \& Torrie, 1980). Significance was established at $P<0.05$.

\section{Results and discussion}

\section{Lipid peroxidation during fermentation and accompanying changes in the rate of expansion}

After 3\% linoleic acid was added to the other bread ingredients, the doughs with and without lipoxygenase were individually mixed with a mixer for a fixed time. Next, the dough underwent primary fermentation in an incubator for $90 \mathrm{~min}$, and the amount of hydroperoxide produced over this time period was measured. These results are shown in Fig. 1. Lipid peroxidation by lipoxygenase induction increased as the fermentation time progressed. However, the overall amount of hydroperoxide produced in the lipoxygenasefree dough tended not to increase. The rate of expansion during this time is shown in Fig. 2.

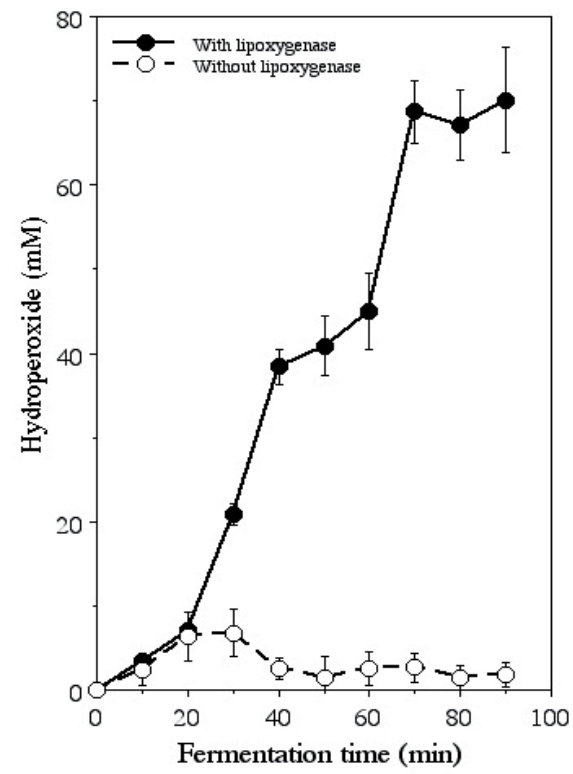

Figure 1. Changes in the amount of hydroperoxide produced with the fermentation of dough. Each value represents the mean \pm standard error in triplicate. 
For up to $40 \mathrm{~min}$, dough with lipoxygenase expanded rapidly after the start of fermentation, but after this time the expansion tended to decrease abruptly. In contrast, lipoxygenase-free dough reached its maximum rate of expansion in $30 \mathrm{~min}$ from the start of fermentation, and this tended to decrease gradually afterwards. Further, in the dough without lipoxygenase, changes in the rate of expansion per unit time were smaller than in the dough with lipoxygenase. Changes brought about by this phenomenon are quite likely due to the effect that hydroperoxide, which is produced by lipid peroxidation, has on the fermentation stage.

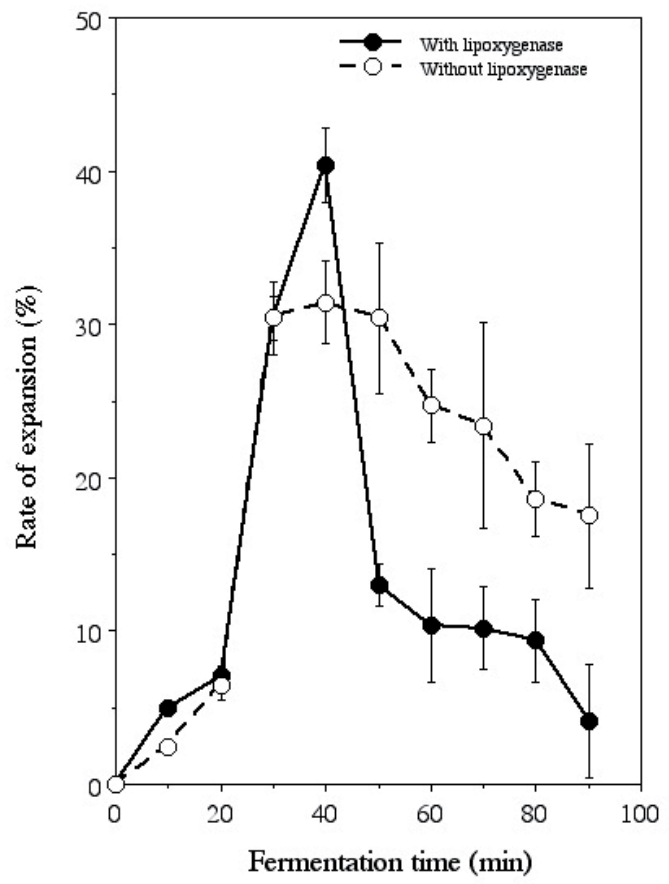

Figure 2. Changes in the rate of expansion with the fermentation of dough. Each value represents the mean \pm standard error in triplicate.

\section{Relationship between the rate of expansion and hydroperoxide}

A model system of gluten and linoleic acid was created, and the involvement of the hydroperoxide that was produced in the fermentation of dough was examined. These results are shown in Fig. 3. The rate of dough expansion was affected by hydroperoxide concentration, and in this experiment the rate of expansion reached its maximum at a hydroperoxide concentration of $30-40 \mathrm{mM}$. Based on these results, the fermentation of dough was influenced by the concentrations of hydroperoxide that were produced. 


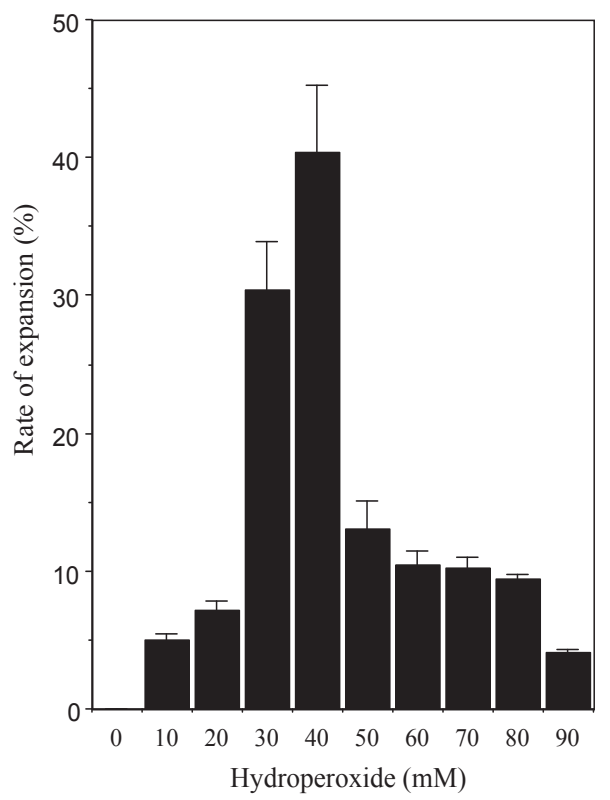

Figure 3. Changes in the rate of dough expansion with hydroperoxides. Each value represents the mean \pm standard error in triplicate.

\section{Effects of yeast and gluten during fermentation}

The effects of the yeast on dough fermentation were also studied. The comparison of the dough with and without lipoxygenase is shown in Fig. 4 . Both doughs with $\leq 2.0 \%$ yeast had similar rates of expansion that tended to increase with fermentation time. There were almost no changes in the rate of expansion with yeast concentrations of $\geq 2.5 \%$; in fact, the rate of expansion tended to decrease. However, the rate of expansion of dough with lipoxygenase tended to increase more than the rate of the dough without lipoxygenase. Since a detailed study of the relationship between yeast and hydroperoxide produced was not done in this experiment, further study is needed.

Next, changes in the amount of hydroperoxide were studied; these results are shown in Fig. 5. For the dough with lipoxygenase, the amount of hydroperoxide reached its maximum when the yeast concentration was $1 \%$; as the yeast concentration increased, the amount of hydroperoxide that was produced tended to decrease. Comparing these results with those in Fig. 4 indicates that there is a relationship between the amount of hydroperoxide produced and the yeast concentration; the specifics of this relationship need to be further investigated. Lipoxygenase-free dough produced almost no hydroperoxide. However, based on the results in Fig. 4, the production of hydroperoxide may not be the sole factor involved in the fermentation of dough. Thus, the hydroperoxide that is produced may be synergistically involved in the mechanism of yeast fermentation. 


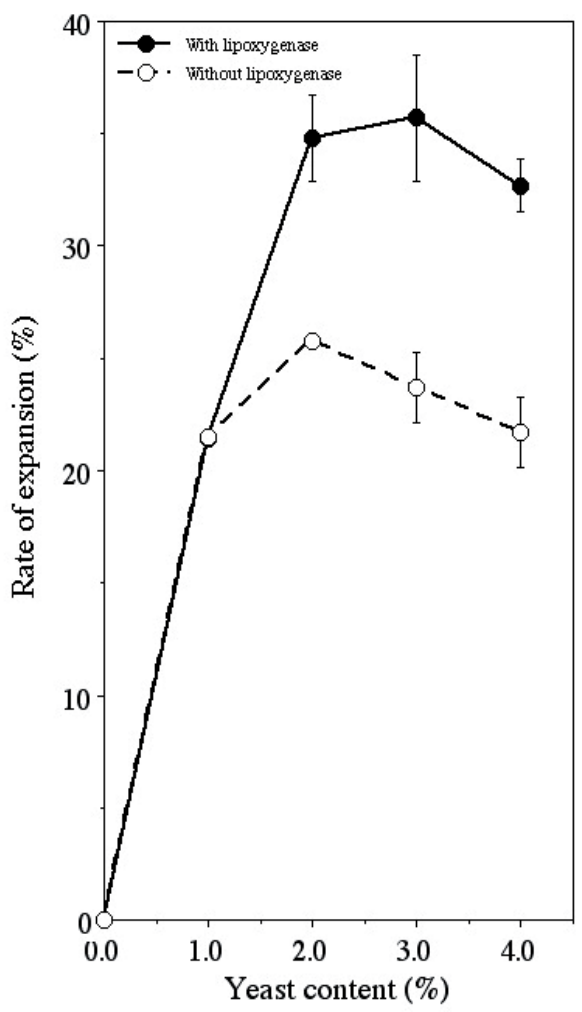

Figure 4. Effect of yeast contents on the rate of dough expansion. Each value represents the mean \pm standard error in triplicate.

Next, the effect of differences in gluten content on dough fermentation was studied. The results are shown in Fig. 6. The expansion of dough began at a gluten content of $40 \%$, and the rate of expansion reached its maximum at a gluten content of $60 \%$. Beyond this concentration, the rate of expansion tended to gradually decrease. Dough with lipoxygenase had a rate of expansion of about $35 \%$ at a gluten content of $60 \%$, while lipoxygenase-free dough had a rate of expansion of $15 \%$. Thus, the presence of hydroperoxide had an effect on the rate of expansion; the hydroperoxide that was produced promoted fermentation.

\section{The relationship between gluten and hydroperoxide}

Hydroperoxide is involved in the fermentation of dough in a facilitatory manner, and, as a result, the rate of dough expansion is increased. Consequently, this phenomenon has a positive effect on dough. To study the effect of the hydroperoxide that is produced during dough fermentation on gluten, the gluten was separated and purified after the completion of fermentation using affinity chromatography, so that, ultimately, the gluten fraction was 
obtained. This gluten fraction was subjected to SDS-gel electrophoresis, and the relationship between gluten and hydroperoxide in the fermentation stage was studied; the results are shown in Fig. 7. In the dough without lipoxygenase, there were almost no changes in the molecular weight of gluten during $100 \mathrm{~min}$ of fermentation time. In contrast, in the dough with lipoxygenase, changes in the molecular weight of gluten were seen with fermentation, and formation of gluten polymers was noted with fermentation. This phenomenon was caused by hydroperoxide that was produced, which acted on the gluten and may have induced denaturation. A comparison of the results shown in Figure 4 and 5 shows that the gluten network was tightened, because the hydroperoxide that was produced by the addition of lipoxygenase denatured the gluten and, subsequently, increased dough expansion.

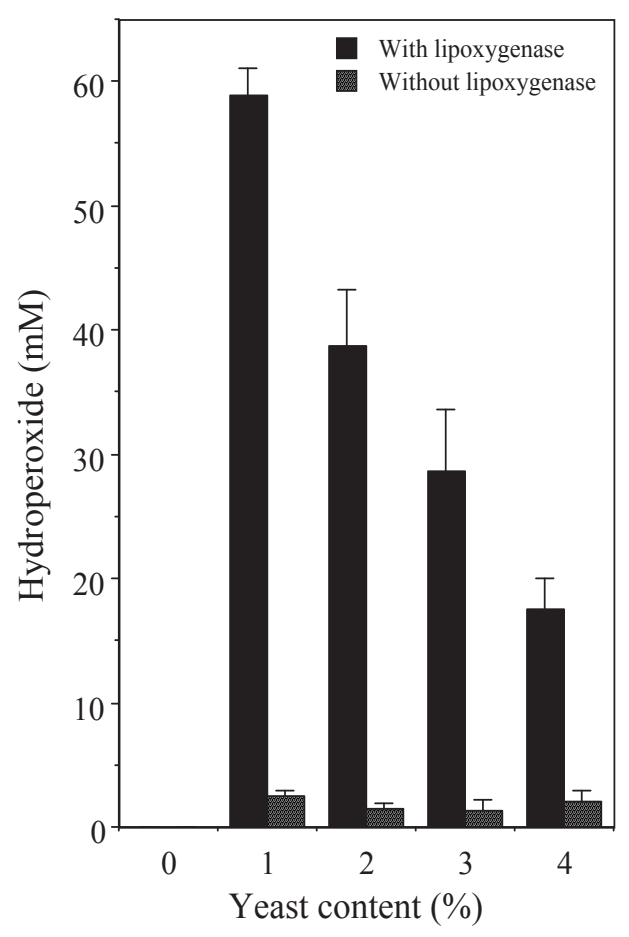

Figure 5. Effect of yeast contents on the amount of hydroperoxide produced in dough. Each value represents the mean \pm standard error in triplicate. 


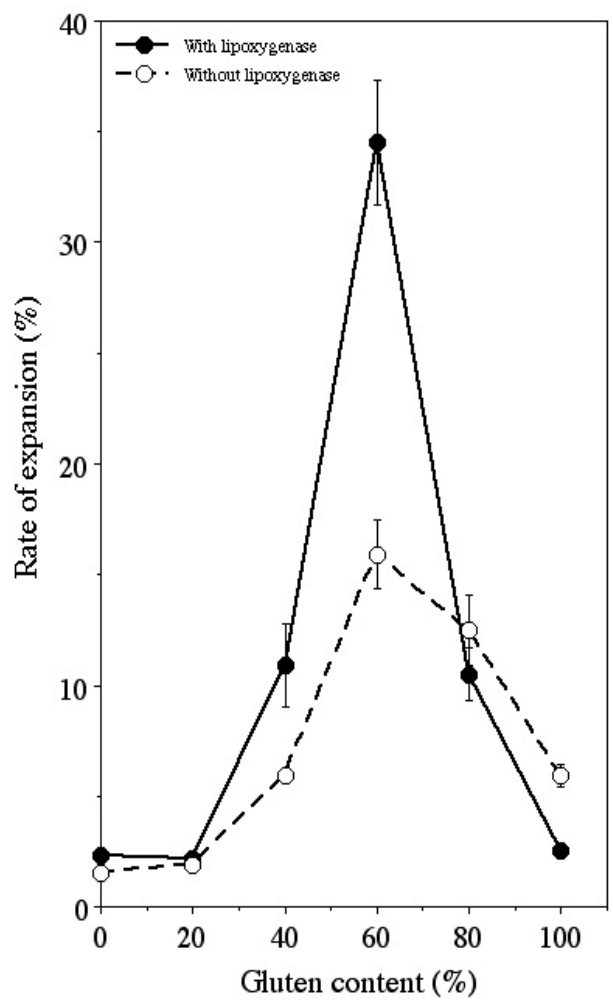

Figure 6. Effect of gluten content on the rate of dough expansion. Each value represents the mean \pm standard error in triplicate.

Without Lipoxygenase

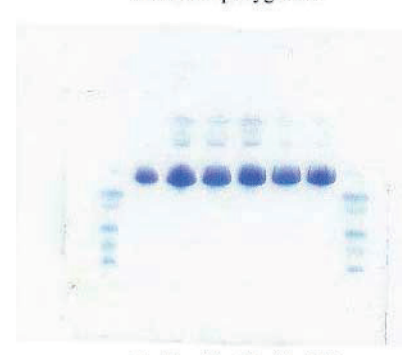

$\begin{array}{llllll}0 & 20 & 40 & 60 & 80 & 100\end{array}$

Fermentation time (min)
With Lipoxygenase

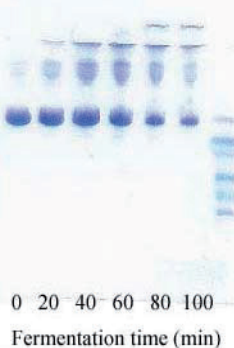

Figure 7. Changes in the molecular weight of gluten when dough was fermented for $100 \mathrm{~min}$. 


\section{The mechanism by which hydroperoxide acts to promote fermentation}

The various experimental results that were obtained were comprehensively analyzed to determine the mechanism of action by which hydroperoxide acts to promote fermentation; this is shown in Fig. 8. During gluten formation, gluten is formed when gliadin and glutenin form a network structure. When gluten is crosslinked in the presence of hydroperoxide, the molecules themselves form macromolecules. As a result, expansion is promoted by the uptake of large amounts of carbon dioxide gas produced during dough fermentation. This phenomenon is ultimately advantageous when baking dough, and it improves the bread's texture. When very little lipid peroxidation is induced, the unoxidized linoleic acid has no interaction with gluten, and, as a result, gluten crosslinking does not occur, which results in baked bread with a poor texture.

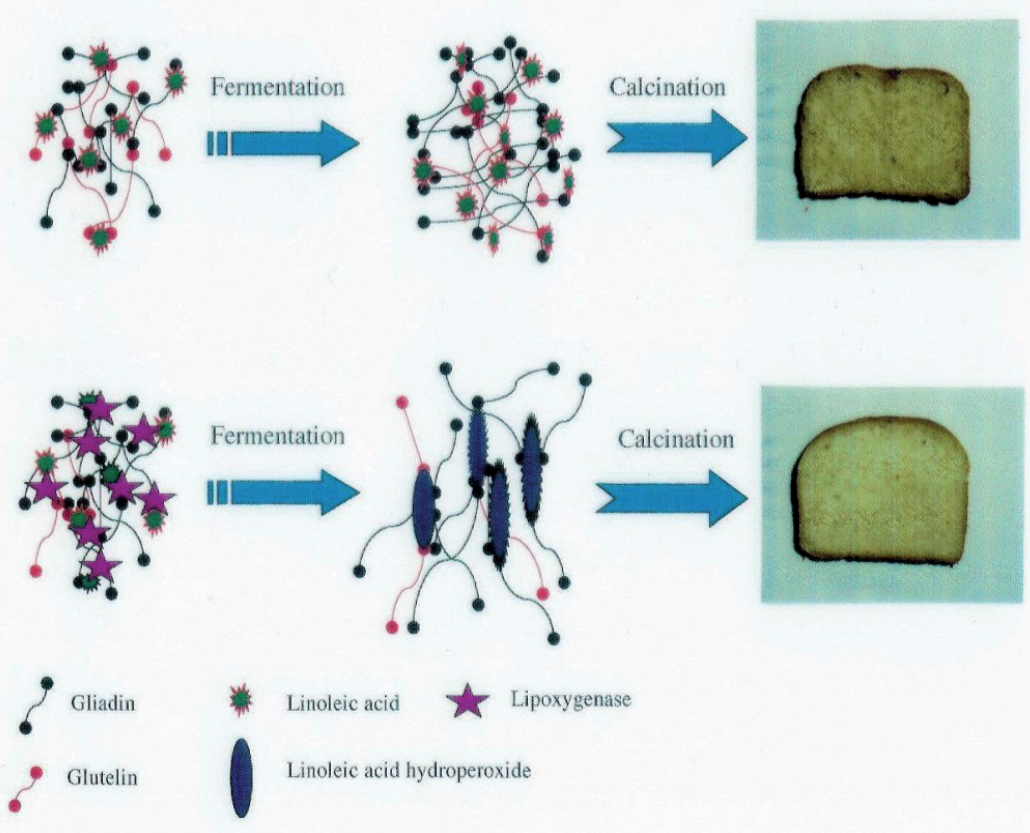

Figure 8. The mechanism by which hydroperoxides accelerate fermentation.

\section{Conclusions}

The current research demonstrated that well-fermented dough can be produced by the induction of lipid peroxidation when fermenting dough. The induction of lipid peroxidation was achieved in the current study by using lipoxygenase induction, but a similar phenomenon should also occur with lipid peroxidation induced by other methods. This phenomenon is advantageous when baking bread and can be used to enhance the quality of 
baked bread. Based on the results of these tests of physical properties, further detailed study is needed of the effect of lipid peroxidation on the flavor of baked bread.

\section{Author details}

Toshiyuki Toyosaki

Department of Foods and Nutrition, Koran Women's Junior College, Fukuoka, Japan

\section{References}

[1] Ames, B.M., Shigena, K., \& Hagen, T.M. (1994). Oxidants, antioxidants and the degenerative diseases of aging. Proceeding National Academy Science, 90, 7915-7922.

[2] Cathcart, R., Schwiers, E., \& Ames, N. (1984). Detection of picomole levels of lipid hydroperoxides using a dichloro-fluorescein fluorescent assay. In L. Packer (Ed), Methods in Enzymology, vol. 105 (p. 352). New York; Academic Press Inc.

[3] Gardnaer, H.W. (1996). Lipoxygenase as a versatile biocatalyst. Journal of American Oil Chemical Society, 73, 1347-1350.

[4] Grosch, W. (1987). Reactions of hydroperoxides-products of low molecular weight. In H.W.-S Chan (Ed), Autoxidation of Unsaturated Lipids. London; Academic Press Inc.

[5] Laemmli, U.K. (1979). Cleavage of structural proteins during the assembly of the head of bacteriophage T4. Nature, 227, 680-685.

[6] Lowry, O.H., Rosebrough, N.J., Farr A.L., \& Randall, R.J. (1951). Protein measurement with the Folin-Phenol reagent. Journal of Biological Chemistry, 193, 265-275.

[7] Mercier, M., \& Gelinas, P. (2001). Effect of lipid oxidation on dough bleaching. Cereal Chemistry, 78, 36-38.

[8] Pokorny, J. (1999). Handbook of Food Preservation, Antioxidants in Food Preservation. New York; Marcel Dekker.

[9] Shewfelt, R.L., \& Del Rosario, B.A. (2000). The role of lipid peroxidation in storage disorders of fresh fruits and vegetables. Horticulture Science, 35, 575-579.

[10] Steel, R.G.D., \& Torrie, J.H.(1980). Principles and procedures of statistics: a biometrical approach (2nd ed). New York: McGraw-Hill.

[11] Toyosaki, T., \& Sakane, Y. (2002). Antioxidant effect of $\mathrm{NaCl}$ on the aqueous solution, emulsified and enzymic lipid peroxidation. Bulletin of the Society of Sea Water Science, Japan, 56, 10-16.

[12] Toyosaki, T., \& Koketsu, M. (2004). Oxidative stability of silky fowl eggs. Comparison with hen eggs. Journal of Agriculture and Food Chemistry, 52, 1328-1330. 